Session 2263

\title{
On the Integration of Fluid Flows and Fabrication
}

\author{
John E. Matsson
}

Oral Roberts University

\begin{abstract}
This paper shows the integration of fabrication and fluid mechanics. The project consists of the design and fabrication of a portable Taylor-Couette apparatus for visualization of centrifugal instabilities in fluid flows. Students were told to design the apparatus so that it would show both primary and secondary instabilities. This necessitated incorporating calculations of the relevant parameter regions and the determination of a suitable method for visualization of the flow. Furthermore, the students had to choose appropriate materials, optimize production cost, determine fabrication techniques for the apparatus, and implement this in the workshop. The final result was a useful piece of equipment for studies of fluid flow instabilities.
\end{abstract}

\section{Introduction}

In this study, an apparatus was constructed in the undergraduate program manufacturing processes course, and used for lab and demonstration purposes in fluid mechanics.

The apparatus consists of two independently rotating, vertical cylinders with a fluid in between the cylinders. The students use the apparatus in the case of a fixed outer cylinder to determine the critical Taylor number ${ }^{1}$. This is a non-dimensional flow parameter defined as $T a=\frac{r_{i}\left(r_{o}-r_{i}\right)^{3} \Omega_{i}^{2}}{v^{2}}$, where $\Omega_{i}$ is the angular velocity of the inner cylinder, $v$ is viscosity of the fluid, and $r_{i}, r_{o}$ are the radii of the inner and outer cylinder, respectively. Visualization of the flow was made by mixing the fluid (silicone oil) with a small amount (less than $0.1 \%$ by weight) of titanium - dioxide coated platelets (10-20 microns in diameter and 3-4 microns in thickness). Such platelets will become oriented by the flow ${ }^{2}$. By using this visualization technique, the critical Taylor number, $T a_{c}=1780^{1}$, determined from linear stability calculations can be compared with

\footnotetext{
${ }^{1}$ The critical Taylor number is increasing as the radius ratio $\eta=r_{i} / r_{o}$ is decreasing. For the present experimental set up the radius ratio $\eta=0.93$.

Proceedings of the 2002 American Society for Engineering Education Annual Conference \& Exposition Copyright (C) 2002, American Society for Engineering Education
} 
the experiments. Above the critical value, the basic flow is replaced by a primary instability in the form of three-dimensional flow pattern consisting of counter-rotating axial-symmetric vortices with a certain non-dimensional span-wise wave number $\alpha=2 \pi\left(r_{o}-r_{i}\right) / \lambda$. The critical span-wise wave number is $\alpha_{c}=3.128$ for the radius ratio $\eta=0.93$, i.e. the span-wise wave-length is almost equal to twice the distance between the cylinders. The linear stability theory is based on an infinite span-wise aspect ratio $\Gamma=L /\left(r_{o}-r_{i}\right)$, where $L$ is the length of the cylinders (present experiments $\Gamma=190$ ).

The earliest experiments on this so called Taylor-Couette flow were made in order to determine the viscosity of various fluids ${ }^{3,4}$. At Taylor numbers slightly higher than the critical one, a secondary instability appears in the form of wavy Taylor vortices, i.e. there exists a periodicity in the circumferential direction.

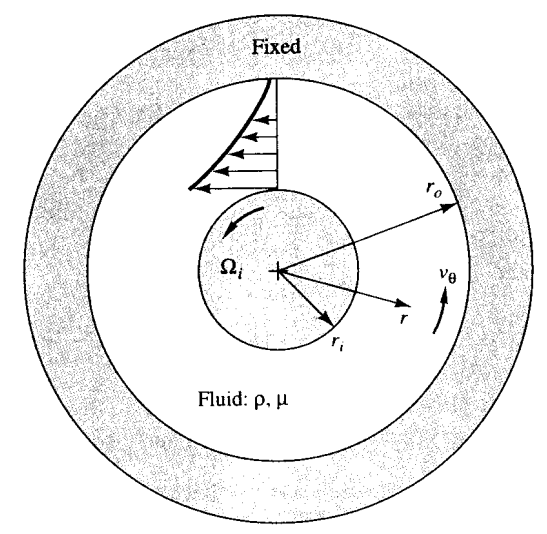

Figure 1. A sketch of the geometry and basic velocity profile for Taylor-Couette flow between two vertical cylinders with a fixed outer cylinder.

The apparatus can also be positioned horizontally. The flow between two horizontal and partially filled rotating cylinders is called Taylor-Dean flow ${ }^{5}$. Linear stability calculations of this flow case show that the critical Taylor number can be dramatically increased for certain parameter combinations. When the Taylor number is increased, the neutral stability curves can consist of two branches that are equally unstable. In this region no stationary instability exists. The instability in this region sets in as a non-axial-symmetric oscillatory solution in the form of traveling waves ${ }^{6}$.

\section{Construction}

The first step in the construction process was selecting the materials to build the apparatus. Most of the materials in the construction were chosen due to their low cost and availability.

After the materials were selected, a design was created and drawn in the engineering graphics software SolidWorks ${ }^{7}$, see Figure 2. One of the design and construction challenges for the students was to keep the fluid contained between the two acrylic cylinders. The plugs for the cylinders were machined to accept the axial shaft and sealed using $o$-rings. The ends of the cylinders were also machined to accept the plugs using a 


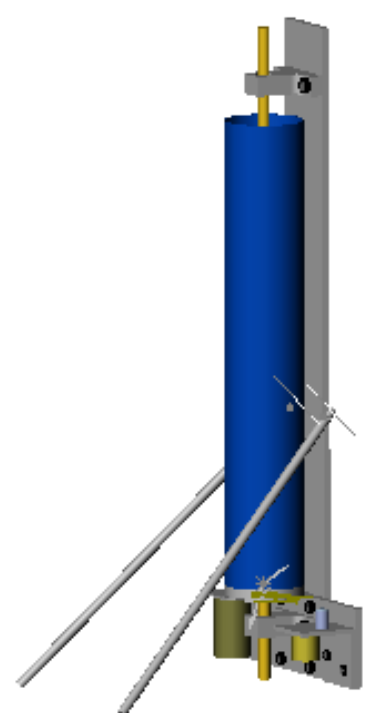

a)

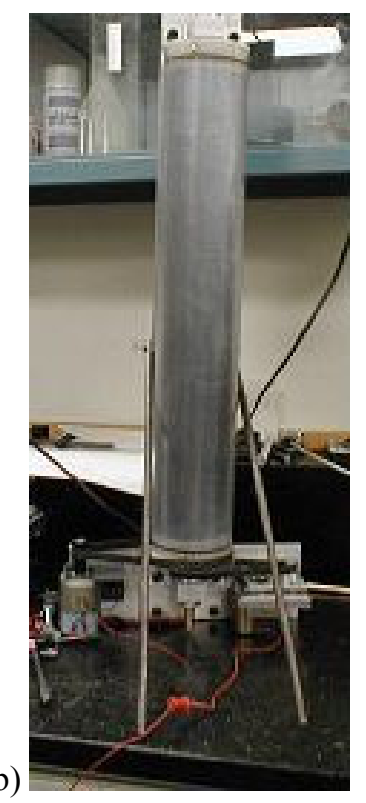

Figure 2. a) SolidWorks model of the experimental apparatus, b) photograph of the final setup.

special carriage mounted on the lathe. The axial shaft was constructed by turning down a solid brass block on the lathe. The support bearing blocks were made from aluminum that was machined on a milling machine. After the bearing blocks were made, the roller bearings were pressed into their final position. The bearing blocks and the tube assembly were then mounted on an aluminum frame.

The aluminum frame consists of two pieces of aluminum plate. The plate was cut using a band saw. Holes were drilled in proper positions for the mounting brackets and motor mounts. Vertical stabilizer steel rods were also mounted on the sides of the frame.

\section{Experimental Results}

The experiments consisted of determining the critical Taylor number and spanwise wave number for Taylor-Couette flow with a fixed outer cylinder. Figure 2a) shows flow visualization of a subcritical flow $(T a=1610)$. At a higher supercritical flow ( $T a=$ 1950), weak axisymmetric vortices are visible with a spanwise wave number $\alpha=2.88$. For an even higher Taylor number $(T a=2545)$, the vortices are stronger and the wave number has increased to $\alpha=2.96$. At the highest Taylor number shown $(T a=4570)$, the spanwise wave number has decreased to $\alpha=2.66$. 


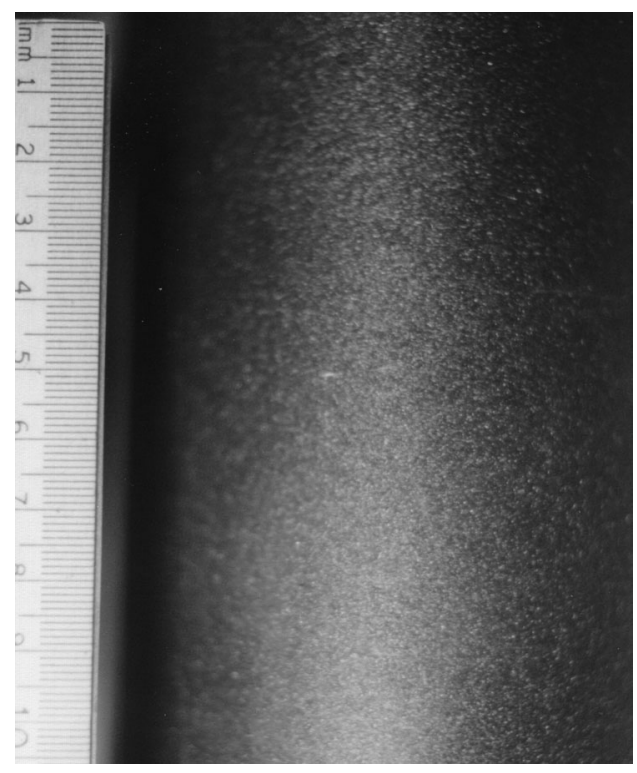

a)
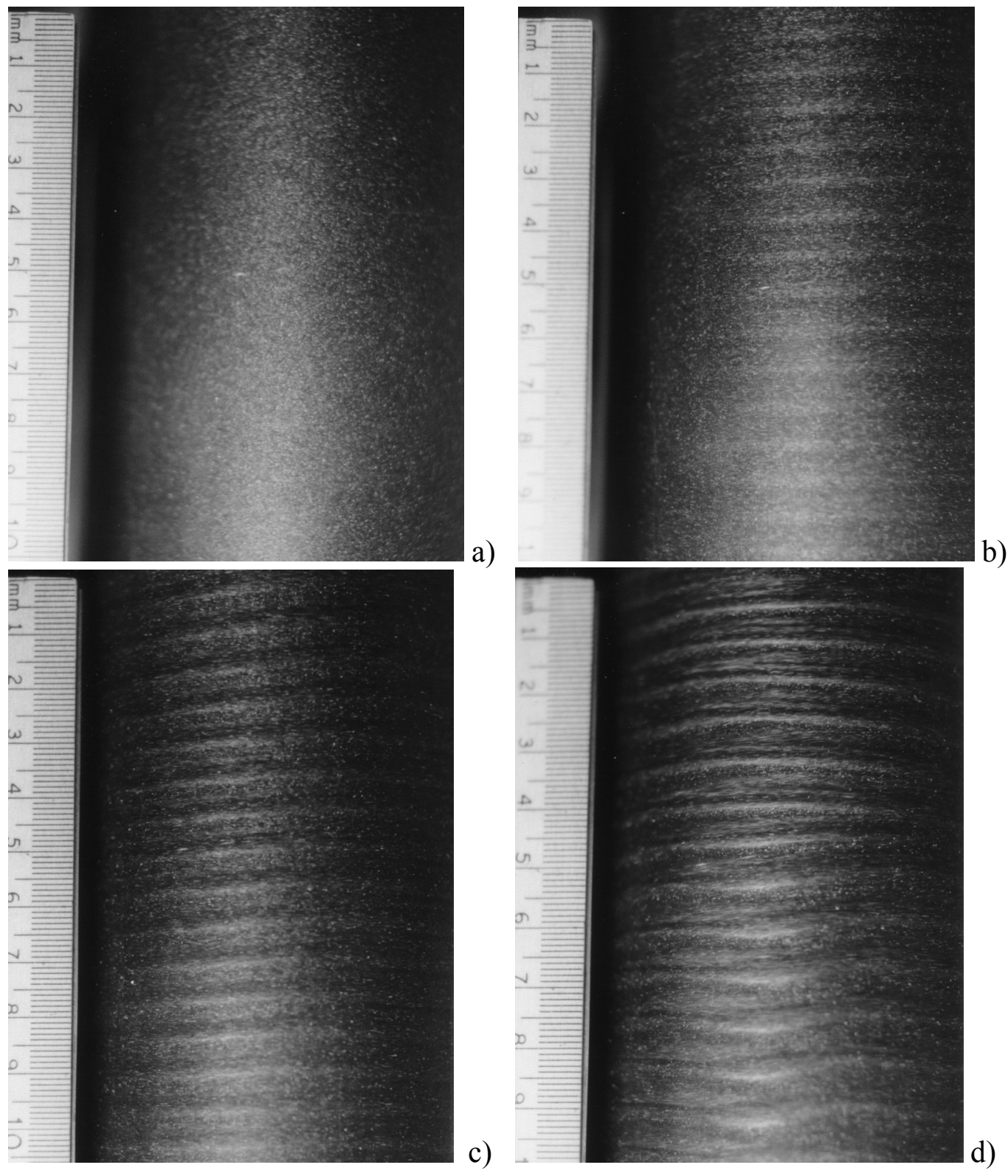

Figure 2. Flow visualization of Taylor-Couette flow with a fixed outer cylinder. a) $T a=1610$, b) $T a=1950$, c) $T a=2545$, d) $T a=4570$.

Furthermore, the vortices have a clear waviness in the lower part of the photograph, see Figure $2 \mathrm{~d}$ ). The results from the experiments were compared with the neutral stability curve $^{8}$ to the first approximation in the narrow gap case $(\eta \rightarrow 1)$ given by

$$
T a=\frac{\left(\pi^{2}+\alpha^{2}\right)^{3}}{\alpha^{2}\left\{1-16 \alpha \pi^{2} \cosh ^{2}(\alpha / 2) /\left[\left(\pi^{2}+\alpha^{2}\right)^{2}(\sinh \alpha+\alpha)\right]\right\}}
$$




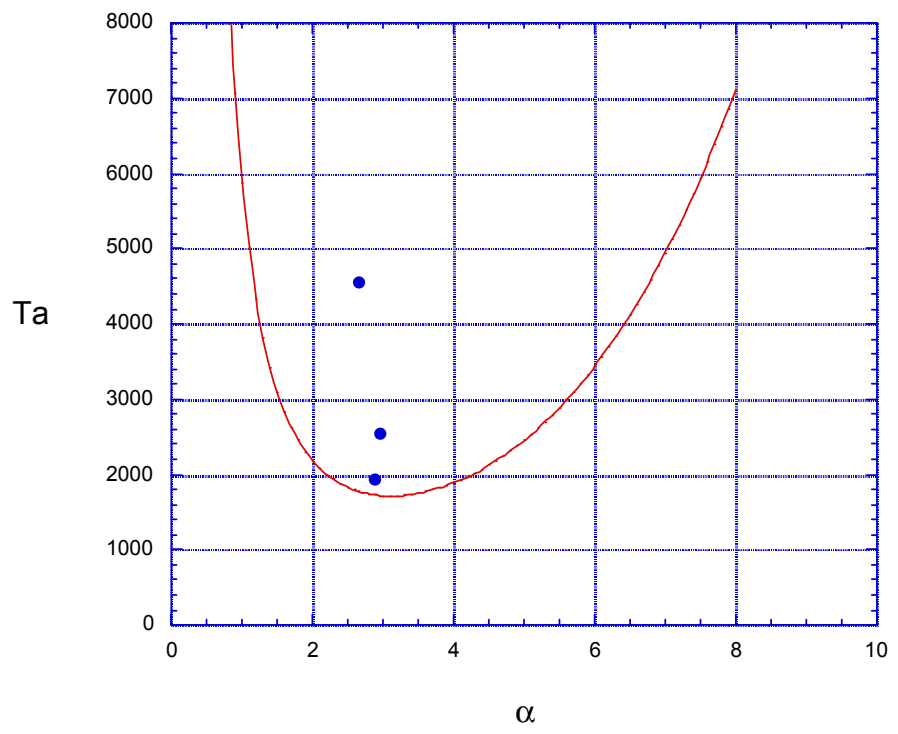

Figure 3. A comparison between experimental results and the neutral stability curve of Taylor-Couette flow with a fixed outer cylinder.

\section{Conclusion}

This paper has shown a project done by undergraduate students in mechanical engineering. It was initiated as a design project in manufacturing processes where the students designed an apparatus for visualization of fluid flow instabilities caused by centrifugal effects.

The apparatus was then used in a fluid mechanics lab where different flow patterns were studied. It was found by flow visualizations that the critical Reynolds number was in the range $T a_{c}=1610-1950$, which is in agreement with linear stability theory that predicts $T a_{c}=1780$.

\section{Bibliography}

1. $\quad$ Taylor G. I., "Stability of a Viscous Liquid Contained between Two Rotating Cylinders." Philos. Trans. Roy. Soc. London Ser. A, vol. 223, (1923).

2. $\quad$ Savas, Ö. On flow visualization using reflective flakes. J. Fluid Mech. 152, 235 (1985).

3. $\quad$ Couette, M. "Etudes sur le frottement des liquides."Ann. Chim. Phys. 21, 433-510 (1890)

4. $\quad$ Mallock, A., "Experiments on fluid viscocity.", Phil. Trans. Roy. Soc. A 187, 41-56 (1896)

5. DiPrima R.C., "The stability of viscous flow between rotating concentric cylinders with a pressure gradient acting round the cylinders."J. Fluid Mech. 6, 462-468 (1959).

6. Mutabazi et al,"Oscillatory modes in the flow between two horizontal corotating cylinders with a partially filled gap. Phys. Rev. A. 39, 763-771 (1989)

7. Planchard M.P. and Planchard D.C., "Engineering Design with SolidWorks 2001." SDC Publications

8. Chandrasekhar, S., "Hydrodynamic and Hydromagnetic Stability". Dover (1981). 